\title{
Long-term changes in sleep duration, energy balance and risk of type 2 diabetes
}

\author{
Elizabeth M. Cespedes ${ }^{1,2,3} \cdot$ Shilpa N. Bhupathiraju ${ }^{1}$ - Yanping Li $^{1}$ • Bernard Rosner ${ }^{4,5}$. \\ Susan Redline ${ }^{6,2} \cdot$ Frank B. Hu ${ }^{1,2}$
}

Received: 24 June 2015 / Accepted: 21 September 2015 / Published online: 2 November 2015

(C) Springer-Verlag Berlin Heidelberg 2015

\begin{abstract}
Aims/hypothesis Baseline sleep duration has a U-shaped relationship with type 2 diabetes, but little research examines the associated changes. We examined long-term changes in sleep duration and concomitant changes in diet, physical activity, weight and subsequent diabetes.

Methods The cohort includes 59,031 women aged 55-83 years in the Nurses' Health Study without diabetes in 2000. Change in sleep duration is the difference between self-reported $24 \mathrm{~h}$ sleep duration in 1986 and 2000. Diet, physical activity and covariates were updated every $2-4$ years. Self-reported diabetes was confirmed via validated questionnaires. Cox regression models were adjusted for 1986 sleep duration and 1986 values of diabetes risk factors, including BMI, and subsequently for change in covariates from 1986 to 2000 .

Results We documented 3,513 incident diabetes cases through to 2012. Compared with no change, decreases in
\end{abstract}

Elizabeth M. Cespedes

emc611@mail.harvard.edu

Department of Nutrition, Harvard T.H. Chan School of Public Health, Boston, MA, USA

2 Department of Epidemiology, Harvard T.H. Chan School of Public Health, Boston, MA, USA

3 Division of Research, Kaiser Permanente Northern California, 2000 Broadway, 5th Floor, Oakland, CA 94612, USA

4 Channing Division of Network Medicine, Harvard Medical School, Boston, MA, USA

5 Department of Biostatistics, Harvard T.H. Chan School of Public Health, Boston, MA, USA

6 Division of Sleep and Circadian Disorders, Departments of Medicine and Neurology, Brigham and Women's Hospital, Boston, MA, USA sleep duration were adversely associated with changes in diet quality and physical activity, while increases were associated with greater weight gain. After adjustment for 1986 covariates, HRs $(95 \% \mathrm{CI})$ for $\leq-2,>-2$ to $<0,>0$ to $<2$ and $\geq 2 \mathrm{~h} /$ day changes in sleep duration (vs no change) were 1.09 ( 0.93 , 1.28), 1.10 (1.001, 1.12), $1.09(1.00,1.18)$ and 1.30 (1.14, 1.46), respectively. Additional adjustment for diet and physical activity did not appreciably alter the results. Increases in sleep duration $\geq 2 \mathrm{~h} /$ day remained adversely associated with diabetes (HR [95\% CI]: 1.15 [1.01, 1.30]) after adjustment for change in covariates, including BMI.

Conclusions/interpretation Increases in sleep duration among middle-aged and older women were modestly associated with risk of diabetes; changes in diet, physical activity and BMI did not explain associations.

Keywords Body weight · Diabetes mellitus · Diet · Sleep

\author{
Abbreviations \\ AHEI-2010 Alternate Healthy Eating Index 2010 \\ MET Metabolic equivalent task \\ NHS Nurses' Health Study
}

\section{Introduction}

With 592 million cases projected by 2035, identifying modifiable risk factors for type 2 diabetes is of urgent public health importance [1]. Sleep duration has emerged as a novel prevention target [2]. A dose-response meta-analysis illustrated a $\mathrm{U}$-shaped relationship, with the lowest diabetes risk at $7-8 \mathrm{~h} /$ day [3]. Shortened sleep is related to glucose intolerance, insulin resistance and reduced acute insulin response to glucose [4-7]. For long sleep, some authors suggest that excessive 
time in bed has detrimental effects on health [8], while others argue long sleep is confounded by mood disorders, sleep apnoea and chronic illness [9]. The mechanisms underlying the sleep-diabetes relationship remain unsettled, as does the degree of mediation by lifestyle. Extreme sleep durations may influence both energy intake and expenditure [10]. Experimentally shortened sleep alters appetite-regulating hormones (decreased leptin; increased ghrelin) leading to overeating, increased hunger [11-13], decreased self-regulation and greater sensitivity to food reward $[14,15]$. With respect to energy expenditure, results are inconsistent: limited experimental evidence does not support a substantial impact of sleep restriction on daily energy expenditure $[13,16]$, despite increased fatigue and reductions in reported physical activity [10].

Most epidemiological studies use single, baseline measurements of sleep duration and do not examine whether diet and physical activity explain the sleep-diabetes association. Our study builds on a 2003 publication from the Nurses' Health Study (NHS) in which extreme sleep durations were associated with diabetes before BMI adjustment. [17] In 2003, repeated measures of sleep duration were not yet available; thus, change was not assessed. Our study newly assesses whether long-term (14 year) changes in self-reported sleep duration in the NHS are predictive of concomitant changes in energy balance factors (diet quality, physical activity and weight) as well as subsequent diabetes risk.

\section{Methods}

Population The NHS was initiated in 1976 when 121,700 female registered nurses aged 30-55 years completed a mailed questionnaire. The cohort is followed via biennial questionnaires, as described previously [18, 19]. Among 119,142 active participants in 2000, we excluded women with diabetes prior to $2000(n=11,729)$ or missing responses to questions on habitual sleep duration in $1986(n=34,479)$ or $2000(n=13,903)$, leaving 59,031 women. The Institutional Review Boards at the Harvard T.H. Chan School of Public Health and Brigham and Women's Hospital approved the study protocol.

Type 2 diabetes Cases were defined as self-reported diabetes confirmed via a validated supplementary questionnaire regarding symptoms, tests and hypoglycaemic therapy. Before 1998, we used the National Diabetes Data Group diagnostic criteria. After 1998, we used the ADA criteria. A previous validation showed self-reported diabetes diagnosis through supplemental questionnaire confirmation was highly accurate: of 62 cases confirmed by questionnaire, 61 (98\%) were reconfirmed by medical records [20].

Sleep variables Nurses reported sleep duration in the 1986 and 2000 questionnaires, corresponding to hours of sleep in a typical $24 \mathrm{~h}$ period; response options were $\leq 5,6,7,8,9,10$ or $\geq 11 \mathrm{~h}$, treated continuously with values assigned to the nearest integer $(\leq 5=5 \mathrm{~h} ; \geq 11=11 \mathrm{~h})$. In 1988 , participants reported total years of rotating shifts, characterised as 'at least three nights per month in addition to working days or evenings in that month'. Response options were: never, 1-2, 3-5, 6-9, $10-14,15-19,20-29$ or $\geq 30$ years. Given prior evidence that diabetes risk increases most after $\geq 5$ years of shift work, we categorised this variable $(<5 / \geq 5$ years) [19]. Women reported snoring frequency in 1986, 2000, 2002 and 2008 and physician-diagnosed sleep apnoea in 2008.

Other covariates Information on confounders, e.g. age, race/ethnicity, employment, smoking, menopause, medications (e.g. hormone therapy, antidepressants [yes/no, first measured in 1996] and antihypertensive drugs) and physician diagnoses was collected via biennial questionnaires. Height was self-reported in the first questionnaire and weight in each biennial questionnaire, from which we calculated BMI $\left(\mathrm{kg} / \mathrm{m}^{2}\right)$. A prior validation showed a correlation of 0.96 between self-reported and technician-measured weight [21]. Food and alcohol consumption was collected every 4 years via validated semi-quantitative food frequency questionnaires. Hypertension and hypercholesterolaemia were considered as physician diagnosis or related medication use. Depression was defined as physician-diagnosed depression or antidepressant medication.

Diet quality was assessed by the Alternate Healthy Eating Index (AHEI-2010), based on a high consumption of vegetables, fruit, nuts/legumes, whole grains, long-chain fats, polyunsaturated fatty acids, and low levels of sugar-sweetened beverages and juice, red/processed meat, trans fat and sodium [22]. Higher scores indicated better quality diets. Physical activity was defined from work/leisure activities as weekly energy expenditure in metabolic equivalent (MET)-h (vigorous activity was $\geq 6 \mathrm{METs}$ ) [23]. In a previous validation [24], the correlation of questionnaire-reported physical activity was 0.79 with prospective 1 -week recalls and 0.62 with activity diaries.

Statistical analysis Our main exposure was 14 year change in sleep duration: the difference in $\mathrm{h}$ /day between 2000 and 1986. This continuous change was categorised into a priori groupings: decreases in sleep duration $(\leq-2 \mathrm{~h} /$ day or $>-2$ to $<0 \mathrm{~h} /$ day $)$, no change in sleep between time points $(0 \mathrm{~h} /$ day, reference), and increases in sleep duration ( $\geq 2 \mathrm{~h} /$ day or $>0$ to $<2 \mathrm{~h}$ /day). We computed age-adjusted descriptive statistics by category of change in habitual sleep duration from 1986 to 2000 (Table 1).

We used multivariable linear regression to examine associations of changes in sleep duration (1986-2000) with concomitant changes in energy balance factors over approximately the same period as change in sleep. Change in diet was 
Table 1 Baseline characteristics by change in sleep (1986-2000) in the NHS, $n=59,031$

\begin{tabular}{|c|c|c|c|c|c|c|}
\hline \multirow[t]{2}{*}{ Variable } & \multicolumn{2}{|l|}{ Decrease } & \multirow{2}{*}{$\begin{array}{l}\text { No change } \\
0 \mathrm{~h} / \text { day } \\
41 \% \\
(n=24,349)\end{array}$} & \multicolumn{2}{|l|}{ Increase } & \multirow[t]{2}{*}{$p$ value $^{\mathrm{a}}$} \\
\hline & $\begin{array}{l}\leq 2 \mathrm{~h} / \text { day } \\
5 \% \\
(n=3,170)\end{array}$ & $\begin{array}{l}>-2 \text { to }<0 \text { h/day } \\
21 \% \\
(n=12,626)\end{array}$ & & $\begin{array}{l}>0 \text { to }<2 \text { h/day } \\
25 \% \\
(n=14,494)\end{array}$ & $\begin{array}{l}\geq 2 \mathrm{~h} / \text { day } \\
7 \% \\
(n=4,392)\end{array}$ & \\
\hline Age in $1986^{\mathrm{b}}$ & $51.6(7.5)$ & $51.7(7.4)$ & $51.8(7.0)$ & $52.4(6.7)$ & $53.1(6.6)$ & $<0.0001$ \\
\hline Age in $2000^{\mathrm{b}}$ & $65.6(7.6)$ & $65.7(7.4)$ & $65.8(7.1)$ & $66.4(6.8)$ & $67.1(6.6)$ & $<0.0001$ \\
\hline $\mathrm{BMI}$ in $1986, \mathrm{~kg} / \mathrm{m}^{2}$ & $25.1(4.6)$ & $24.8(4.3)$ & $24.6(4.2)$ & $24.9(4.3)$ & $25.4(4.7)$ & $<0.0001$ \\
\hline BMI in $2000, \mathrm{~kg} / \mathrm{m}^{2}$ & $26.6(5.4)$ & $26.2(4.9)$ & $26.1(4.9)$ & $26.5(5.1)$ & $27.3(5.5)$ & $<0.0001$ \\
\hline Alcohol gm, 1986 & $6.7(11.7)$ & $6.3(10.6)$ & $6.4(10.4)$ & $6.4(10.6)$ & $6.3(11.0)$ & 0.47 \\
\hline Alcohol gm, 2000 & $5.1(9.1)$ & $5.1(9.0)$ & $5.4(9.2)$ & $5.3(9.3)$ & $5.1(10.0)$ & 0.01 \\
\hline Sleep duration, h/day, 1986 & $8.1(0.8)$ & $7.3(1.0)$ & $7.1(0.8)$ & $6.6(0.8)$ & $6.2(0.8)$ & $<0.0001$ \\
\hline Sleep duration, h/day, 2000 & $5.8(0.9)$ & $6.2(1.0)$ & $7.1(0.8)$ & $7.6(0.8)$ & $8.4(0.8)$ & $<0.0001$ \\
\hline AHEI-2010, 1986 & $45.5(10.4)$ & $46.0(10.4)$ & $46.2(10.3)$ & $46.0(10.4)$ & $45.7(10.5)$ & 0.0003 \\
\hline AHEI-2010, 1998 & $48.6(10.1)$ & $49.2(10.1)$ & $49.5(10.0)$ & $49.2(9.8)$ & $49.1(10.0)$ & $<0.0001$ \\
\hline Total activity METS/week, 1986 & $14.0(20.4)$ & $14.5(21.5)$ & $15.0(21.4)$ & $14.7(21.0)$ & $14.1(19.9)$ & 0.01 \\
\hline Total activity METS/week, 2000 & $15.8(22.9)$ & $17.4(21.0)$ & $18.4(21.3)$ & $18.0(22.9)$ & $16.3(25.6)$ & $<0.0001$ \\
\hline Shift work $\geq 5$ years, $\%$ & 18.5 & 15.6 & 14.9 & 16.6 & 20.3 & $<0.0001$ \\
\hline Retired by $2000, \%$ & 65.5 & 63.6 & 64.1 & 67.4 & 73.6 & $<0.0001$ \\
\hline Premenopausal in $1986, \%$ & 35.9 & 35.3 & 35.3 & 34.0 & 32.1 & $<0.0001$ \\
\hline Premenopausal in $2000, \%$ & 1.0 & 1.3 & 1.2 & 1.2 & 1.0 & $<0.0001$ \\
\hline Current smoker in $1986, \%$ & 18.9 & 18.1 & 17.9 & 19.9 & 24.7 & $<0.0001$ \\
\hline Current smoker in $2000, \%$ & 8.2 & 8.0 & 8.3 & 8.8 & 11.3 & $<0.0001$ \\
\hline Quit smoking, 1986-2000, \% & 11.2 & 10.7 & 10.1 & 11.5 & 14.1 & $<0.0001$ \\
\hline Non-Hispanic white, $\%$ & 98.3 & 97.7 & 98.3 & 98.0 & 97.5 & 0.0002 \\
\hline Family history of diabetes, $\%$ & 27.9 & 27.3 & 27.0 & 27.6 & 28.8 & 0.12 \\
\hline High BP 2000, \% & 49.9 & 45.3 & 44.0 & 46.9 & 52.1 & $<0.0001$ \\
\hline High cholesterol 2000, \% & 63.6 & 60.3 & 58.8 & 59.6 & 62.1 & $<0.0001$ \\
\hline Antidepressant use $2000, \%$ & 11.0 & 8.2 & 7.6 & 10.3 & 18.4 & $<0.0001$ \\
\hline \multicolumn{7}{|l|}{ Sleep duration, $\%$} \\
\hline$\leq 6 \mathrm{~h} /$ day in 1986 & 0.0 & 17.3 & 23.6 & 44.1 & 67.7 & $<0.0001$ \\
\hline$\leq 6 \mathrm{~h} /$ day in 2000 & 81.8 & 57.2 & 23.6 & 6.7 & 0.0 & $<0.0001$ \\
\hline$\geq 9 \mathrm{~h} /$ day in 1986 & 25.6 & 7.3 & 2.7 & 1.1 & 0.0 & $<0.0001$ \\
\hline$\geq 9 \mathrm{~h} /$ day in 2000 & 0.2 & 0.7 & 2.7 & 11.2 & 42.2 & $<0.0001$ \\
\hline Lost $>2.3 \mathrm{~kg}$ from ' 86 to $' 00, \%$ & 19.6 & 17.8 & 16.5 & 16.1 & 17.3 & $<0.0001$ \\
\hline Gained $>2.3 \mathrm{~kg}$ from ' 86 to $' 00, \%$ & 61.5 & 60.2 & 61.5 & 63.5 & 65.1 & 0.004 \\
\hline Weight change, $\mathrm{kg}$ from ' 86 to ' 00 & $4.2(8.5)$ & $3.9(7.9)$ & $4.1(7.5)$ & $4.5(7.8)$ & $5.0(8.9)$ & $<0.0001$ \\
\hline AHEI-2010 change from ' 86 to ' 00 & $2.9(10.1)$ & $2.9(9.8)$ & $3.2(9.5)$ & $3.1(9.5)$ & $3.2(10.1)$ & 0.22 \\
\hline MET h/week change from ' 86 to '00 & $1.8(25.9)$ & $2.9(25.1)$ & $3.4(24.9)$ & $3.3(26.5)$ & $2.2(28.5)$ & 0.0002 \\
\hline Frequent snoring, $2000, \%$ & 18.8 & 17.8 & 16.7 & 17.7 & 21.0 & $<0.0001$ \\
\hline Ever-diagnosis of sleep apnoea, $\%$ & 3.5 & 3.3 & 2.6 & 2.9 & 4.1 & $<0.0001$ \\
\hline
\end{tabular}

Values are means (SD) or percentages and are standardised to the age distribution of the study population

${ }^{\mathrm{a}} p$ value from ANOVA or $\chi^{2}$ test

${ }^{\mathrm{b}}$ Values are not age-adjusted

represented by AHEI-2010 (change in score 1986 to 1998, the nearest questionnaire to 2000); physical activity by METS (change in h/week from 1986 to 2000) and energy balance by weight (change in $\mathrm{kg}$ from 1986 to 2000). We adjusted for race/ethnicity (non-Hispanic white, yes/no) and sleep duration at the start of the change period (1986) and covariates, including age (years), retired (yes/no), menopause (premenopausal or hormone therapy [never, past, or current user]), alcohol grams in quartiles, smoking (never, past, or current smoker [1-14, 1524 or $25+$ cigarettes/week]), diabetes family history (yes/no), 
snoring frequency (most nights, some or never), sleep apnoea (ever-diagnosis), antidepressant use (yes/no), shift work history ( $\geq 5$ years), BMI $(<20.9,21-24.9,25-29.9,30.0-31.9,32.0+$ $\mathrm{kg} / \mathrm{m}^{2}$ ), and hypertension or hypercholesterolaemia. Subsequent models adjusted for changes in these covariates (1986-2000).

To examine the primary outcome of diabetes, we used Cox regression models jointly stratified by age in months at the start of follow-up and calendar year of the current questionnaire cycle to estimate HRs and 95\% CI according to category of change in sleep duration. Individuals contributed persontime from the return of the 2000 questionnaire until the date of diagnosis of diabetes, death, loss to follow-up or end of the follow-up period (30 June 2012). Models adjusted for the variables listed above at the start of the change period in 1986 and for change in covariates (1986-2000).

We varied our modelling approach by using an additional categorical exposure: we cross-classified women according to sleep duration in 1986 and 2000 (short [ $\leq 6 \mathrm{~h} /$ day], long [ $\geq 9 \mathrm{~h} /$ day] and normative [7-8 h/day]), with women reporting $7-8 \mathrm{~h} /$ day at both time points as the reference for comparison with women reporting consistently short, consistently long or changes in sleep duration (Fig. 1).

Sensitivity analyses To limit potential reverse causation, we considered excluding cases within the first 2 years of follow-up $(n=758)$ and those with chronic disease in 2000 (ever-diagnosis of cancer and/or cardiovascular disease $[n=11,186])$. We considered excluding women $>66$ years (median age) in 2000 $(n=30,243)$ or who had conducted shift work for $\geq 5$ years $(n=9,503)$.

Data were analysed using SAS for UNIX (version 9.3; SAS Institute, Cary, NC, USA). Statistical significance was set at a two-tailed $p<0.05$.

\section{Results}

Average sleep duration remained constant between 1986 and 2000; half of women (49\%) reported normative durations (7-8 h/night) at both time points, while $15 \%$ were consistently short-sleepers ( $\leq 6 \mathrm{~h}$ ) and $2 \%$ consistently long-sleepers $(\geq 9 \mathrm{~h})$. The correlation between sleep measurements in 1986 and 2000 was $r=0.42$. The mean (SD, range) of change in sleep duration was $0(1,-6$ to 5$) \mathrm{h} /$ day. Over the same period (1986 to 2000), AHEI-2010 increased by 3 points (mean [SD] change, 3 [10]), from 46 to 49 ; physical activity increased by $3 \mathrm{MET}$ h/week (mean [SD] change, 3 [26]), from 15 to 18; and weight increased by $4 \mathrm{~kg}$ (mean [SD] change, 4 [8]). BMI increased from mean (SD) 25 (4) $\mathrm{kg} / \mathrm{m}^{2}$ in 1986 to 26 (5) $\mathrm{kg} / \mathrm{m}^{2}$ in 2000 . Mean age (SD, range) at the start of diabetes follow-up in 2000 was $66(7,53-82)$ years.

Table 1 shows age-adjusted descriptive characteristics at the start of follow-up (2000) by categories of our main exposure, change in sleep duration from 1986 to 2000 . Few women reported dramatic decreases $(5 \%)$ or increases $(7 \%)$ in sleep duration $\geq 2 \mathrm{~h} /$ day. Compared with women with lesser or no changes in sleep duration, women reporting increases or decreases $\geq 2 \mathrm{~h} /$ day had higher indices of body mass, lower indices of physical activity and diet quality, were more likely to snore frequently, smoke, have hypertension, hypercholesterolaemia or a family history of diabetes, take antidepressant medications or have conducted shift work for $\geq 5$ years. Women who reported $\geq 2 \mathrm{~h} /$ day increases were slightly older and gained more weight from 1986 to 2000.

Change in sleep duration and changes in energy balance factors Table 2 shows associations of changes in sleep duration from 1986 to 2000 with concurrent changes in diet quality (AHEI-2010), physical activity (MET h/week) and weight

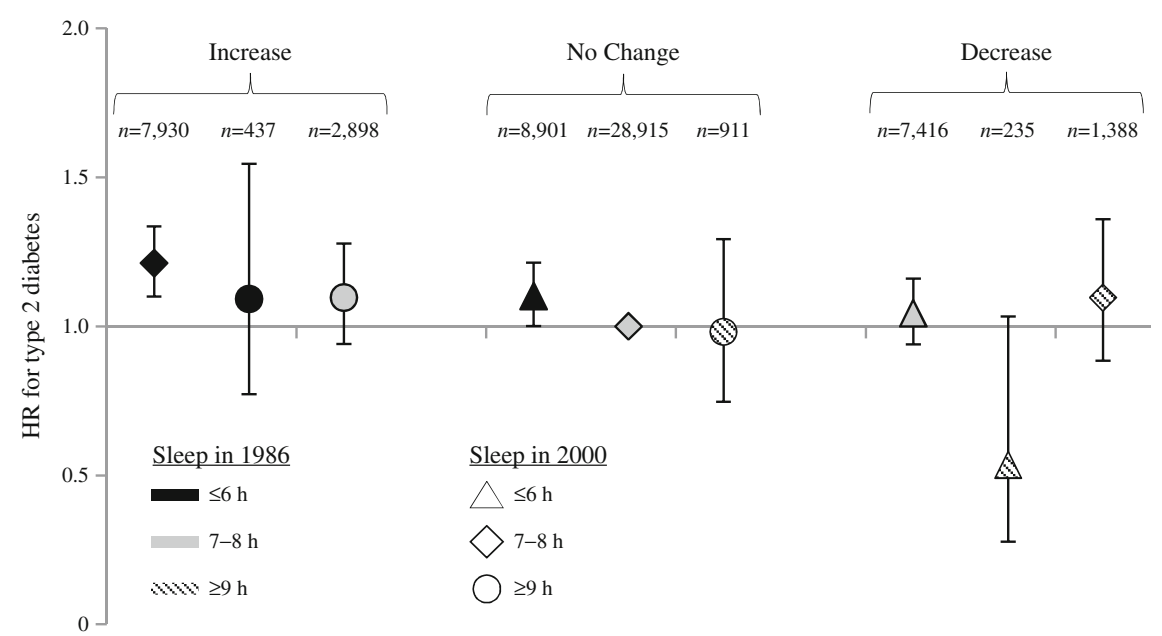

Fig. 1 Change in sleep duration from 1986 to 2000 and risk of type 2 diabetes in the NHS, $n=59,031$. Patterns indicate sleep duration in 1986; shapes indicate sleep duration in 2000 . Consistently short sleep $(\leq 6 \mathrm{~h} /$ day) and short sleep in 1986 increasing to normative sleep (7-8 h/day) in

2000 were adversely associated with diabetes. Models adjust for race/ethnicity, diabetes family history, retirement, shift work and 2000 values of hypercholesterolaemia, hypertension, menopause, sleep apnoea, snoring, depression, smoking and alcohol 
Table 2 Change in sleep duration and change in energy balance factors in the NHS, 1986-2000, $n=59,031$

\begin{tabular}{|c|c|c|c|c|c|c|c|c|c|}
\hline Variable & $\leq-2 \mathrm{~h} /$ day & $p$ value & $>-2$ to $<0 \mathrm{~h} /$ day & $p$ value & 0 h/day & $>0$ to $<2 \mathrm{~h} /$ day & $p$ value & $\geq 2 \mathrm{~h} /$ day & $p$ value \\
\hline \multicolumn{10}{|c|}{ Mean difference in change in AHEI-2010, score: $1986-1998$ (95\% CI), $n=50,462$} \\
\hline Age-adjusted & $-0.30(-0.68,0.09)$ & 0.13 & $-0.27(-0.49,-0.05)$ & 0.02 & 0.00 (Ref.) & $-0.05(-0.27,0.16)$ & 0.61 & $0.07(-0.27,0.40)$ & 0.69 \\
\hline Multivariable-adj. ${ }^{\text {a }}$ & $-0.29(-0.69,0.12)$ & 0.16 & $-0.27(-0.49,-0.04)$ & 0.02 & 0.00 (Ref.) & $-0.07(-0.29,0.15)$ & 0.51 & $0.04(-0.31,0.39)$ & 0.83 \\
\hline+ Change in covariates ${ }^{\mathrm{b}}$ & $-0.26(-0.66,0.14)$ & 0.21 & $-0.25(-0.48,-0.03)$ & 0.03 & 0.00 (Ref.) & $-0.04(-0.26,0.18)$ & 0.70 & $0.11(-0.24,0.46)$ & 0.53 \\
\hline+ Change in $\mathrm{BMI}^{\mathrm{c}}$ & $-0.25(-0.65,0.15)$ & 0.22 & $-0.26(-0.48,-0.03)$ & 0.02 & 0.00 (Ref.) & $-0.02(-0.02,0.20)$ & 0.84 & $0.15(-0.20,0.49)$ & 0.81 \\
\hline \multicolumn{10}{|c|}{ Mean difference in change in METS, h/week: $1986-2000(95 \% \mathrm{CI}), n=58,885$} \\
\hline Age-adjusted & $-1.59(-2.53,-0.64)$ & 0.001 & $-0.56(-1.11,-0.01)$ & 0.04 & 0.00 (Ref.) & $0.06(-0.47,0.58)$ & 0.84 & $-1.21(-2.03,-0.39)$ & 0.004 \\
\hline Multivariable-adj. ${ }^{\text {a }}$ & $-1.50(-2.50,-0.50)$ & 0.003 & $-0.57(-1.13,-0.01)$ & 0.05 & 0.00 (Ref.) & $-0.03(-0.57,0.50)$ & 0.90 & $-1.35(-2.20,-0.49)$ & 0.002 \\
\hline+ Change in covariates ${ }^{\mathrm{b}}$ & $-1.22(-2.21,-0.22)$ & 0.02 & $-0.42(-0.97,0.14)$ & 0.14 & 0.00 (Ref.) & $-0.15(-0.69,0.38)$ & 0.57 & $-1.60(-2.45,-0.74)$ & 0.003 \\
\hline+ Change in $\mathrm{BMI}^{\mathrm{c}}$ & $-1.16(-2.15,-0.16)$ & 0.02 & $-0.43(-0.99,0.13)$ & 0.13 & 0.00 (Ref.) & $0.08(-0.62,0.46)$ & 0.77 & $-1.44(-2.29,-0.58)$ & 0.001 \\
\hline \multicolumn{10}{|c|}{ Mean difference in change in weight $(\mathrm{kg}): 1986-2000(95 \% \mathrm{CI}), n=56,758$} \\
\hline Age-adjusted & $0.14(-0.14,0.43)$ & 0.32 & $-0.11(-0.28,0.05)$ & 0.18 & 0.00 (Ref.) & $0.45(0.30,0.61)$ & $<.0001$ & $0.89(0.64,1.13)$ & $<0.0001$ \\
\hline Multivariable-adj. ${ }^{\text {a }}$ & $0.21(-0.09,0.51)$ & 0.17 & $-0.09(-0.26,0.08)$ & 0.31 & 0.00 (Ref.) & $0.33(0.16,0.49)$ & $<.0001$ & $0.55(0.29,0.81)$ & $<0.0001$ \\
\hline+ Change in covariates ${ }^{\mathrm{b}}$ & $-0.03(-0.33,0.26)$ & 0.82 & $-0.21(-0.38,-0.05)$ & 0.01 & 0.00 (Ref.) & $0.31(0.15,0.47)$ & 0.0002 & $0.47(0.21,0.72)$ & 0.0003 \\
\hline
\end{tabular}

${ }^{\text {a }}$ Adjusted for race/ethnicity (non-Hispanic white or other), diabetes family history, shift work history ( $\geq 5$ years), 1986 values of sleep duration, age, retirement (yes/no), BMI $\left(<20.9,21-24.9,25-29.9,30.0-31.9,32.0+\mathrm{kg} / \mathrm{m}^{2}\right)$, high cholesterol or hypertension (yes/no), menopausal status, sleep apnoea (ever-diagnosis), snoring (most nights, some nights or never), antidepressant use, smoking status, alcohol use, diet quality and physical activity ${ }^{\mathrm{b}}$ Additionally adjusted for change from 1986 to 2000 in values of retirement, high cholesterol or hypertension, menopausal status, snoring, antidepressant use, smoking status, alcohol consumption, and either AHEI-2010 (when MET h/week was the outcome) or MET h/week (when AHEI-2010 was the outcome)

${ }^{\mathrm{c}}$ Additionally adjusted for change in BMI $\left(<20.9,21-24.9,25-29.9,30.0-31.9,32.0+\mathrm{kg} / \mathrm{m}^{2}\right)$ from 1986 to 2000

Adj., adjusted; Ref., reference

$(\mathrm{kg})$. Overall, changes in sleep duration were only modestly associated with changes in energy balance factors: associations were small in magnitude after adjustment for 1986 values of confounders (including BMI and sleep duration) and for change in confounders between 1986 and 2000. For example, $>-2$ to $<0 \mathrm{~h}$ /day decreases had an adverse association with changes in AHEI-2010; the mean difference (95\% CI) for change in AHEI-2010 compared with women reporting no change in sleep duration was $-0.25(-0.48$, $-0.03)$ points. Compared with women reporting no change in sleep duration, both extreme decreases $(\leq-2 \mathrm{~h} /$ day) and increases ( $\geq 2 \mathrm{~h} /$ day) were adversely associated with change in physical activity, with mean differences of $-1.16(-2.15$, $-0.16)$ and $-1.44(-2.29,-0.58)$ MET h/week, respectively. Increases but not decreases in sleep duration were associated with greater weight gain: women reporting increases of $>0$ to $<2$ or $\geq 2 \mathrm{~h}$ /day gained $0.31(0.15,0.47)$ and $0.47(0.21,0.72)$ additional kilograms, respectively, between 1986 and 2000.

\section{Change in sleep duration and risk of type 2 diabetes We} observed 3,513 diabetes cases among 59,031 participants over 612,410 person-years of follow-up. In initial Cox regression models stratified on age and calendar time, there was a U-shaped relationship of changes in sleep duration with diabetes; both decreases and increases were associated with greater diabetes (Table 3). After adjusting for sleep duration in 1986 , the HR $(95 \% \mathrm{CI})$ for participants reporting $\leq-2,>-2$ to $0,0,>0$ to $<2$ or $\geq 2 \mathrm{~h} /$ day changes in sleep duration were 1.18 (1.01, 1.39), $1.13(1.03,1.24), 1.09(1.00,1.19)$ and 1.40
$(1.23,1.58)$, respectively. Additional adjustment (race/ethnicity, diabetes family history, shift work, hypercholesterolaemia, hypertension, menopause, snoring, sleep apnoea, antidepressant use, smoking, alcohol, diet, physical activity and BMI), attenuated associations; only increases in sleep duration remained adversely associated with diabetes $(1.15[1.01,1.30])$. Adjusting for AHEI-2010 and MET h/week did not appreciably alter results, nor did subsequent adjustment for change in confounders and in BMI from 1986 to 2000.

When we cross-classified women according to sleep duration in 1986 and 2000, women with chronic short sleep ( $\leq 6 \mathrm{~h} /$ day at both time points), or who initially reported $\leq 6 \mathrm{~h} /$ day but then increased sleep duration, had modestly elevated risk of diabetes compared with women maintaining a normative sleep duration of 7-8 h/day $(1.10[1.001,1.21]$ and $1.21[1.10,1.34]$, respectively). Associations remained statistically significant after adjustment for confounders (Fig. 1), but after BMI adjustment associations of chronic short sleep attenuated $(1.05[0.96,1.16])$ while those with increases in sleep duration persisted (1.16 $[1.05,1.28])$; data not shown. Importantly, interaction terms were not statistically significant and there were few women with sleep durations $\geq 9 \mathrm{~h} /$ day in 1986 .

Sensitivity analyses In sensitivity analyses, excluding cases within the first 2 years of follow-up or excluding participants who conducted shift work for $\geq 5$ years or with prevalent chronic disease did not alter results. Results were also similar when we excluded participants over the median age (66 years) in 2000 and interaction terms of age and change in sleep 
Table 3 Change in sleep duration and subsequent risk of type 2 diabetes, 1986-2000 in the NHS, $n=59,031$

\begin{tabular}{|c|c|c|c|c|c|c|c|c|c|}
\hline Variable & $\leq-2 \mathrm{~h} /$ day & $p$ value & $>-2$ to $<0 \mathrm{~h} /$ day & $p$ value & $0 \mathrm{~h} /$ day & $>0$ to $<2 \mathrm{~h} /$ day & $p$ value & $\geq 2 \mathrm{~h} /$ day & $p$ value \\
\hline Cases & 190 & & 759 & & 1,334 & 889 & & 341 & \\
\hline Person-years & 32,306 & & 131,534 & & 255,889 & 149,769 & & 42,912 & \\
\hline Adjusted for age \& sleep in $1986^{\mathrm{a}}$ & $1.18(1.01,1.39)$ & 0.04 & $1.13(1.03,1.24)$ & 0.01 & 1.00 (Ref.) & $1.09(1.00,1.19)$ & 0.05 & $1.40(1.23,1.58)$ & $<0.0001$ \\
\hline+ Covariates in $1986^{\mathrm{b}}$ & $1.10(0.94,1.30)$ & 0.23 & $1.10(1.003,1.20)$ & 0.04 & 1.00 (Ref.) & $1.09(1.00,1.19)$ & 0.06 & $1.30(1.15,1.47)$ & $<0.0001$ \\
\hline+ AHEI-2010 score and METs/week in $1986^{\mathrm{c}}$ & $1.09(0.93,1.28)$ & 0.28 & $1.10(1.001,1.20)$ & 0.048 & 1.00 (Ref.) & $1.09(1.00,1.18)$ & 0.06 & $1.30(1.14,1.46)$ & $<0.0001$ \\
\hline$+\mathrm{BMI}$ in $1986^{\mathrm{d}}$ & $1.07(0.91,1.25)$ & 0.44 & $1.09(0.99,1.19)$ & 0.08 & 1.00 (Ref.) & $1.07(0.98,1.16)$ & 0.15 & $1.22(1.08,1.38)$ & 0.002 \\
\hline+ Change in covariates ${ }^{\mathrm{e}}$ & $1.02(0.87,1.20)$ & 0.80 & $1.07(0.98,1.17)$ & 0.14 & 1.00 (Ref.) & $1.05(0.96,1.15)$ & 0.25 & $1.18(1.04,1.33)$ & 0.01 \\
\hline+ Change in $\mathrm{BMI}^{\mathrm{f}}$ & $1.03(0.87,1.20)$ & 0.76 & $1.08(0.98,1.18)$ & 0.10 & 1.00 (Ref.) & $1.04(0.95,1.13)$ & 0.46 & $1.15(1.01,1.30)$ & 0.03 \\
\hline
\end{tabular}

Data are HR $(95 \% \mathrm{CI})$ for type 2 diabetes

${ }^{a}$ Adjusted for categorical sleep duration in $1986(\leq 5,6,7,8,9$ or $\geq 10 \mathrm{~h} /$ day $)$

${ }^{\mathrm{b}}$ Adjusted for race/ethnicity (non-Hispanic white or other), diabetes family history, retirement (yes/no), shift work history ( $\geq 5$ years) and 1986 values of high cholesterol or hypertension (yes/no), menopausal status (premenopausal, post-menopausal current, former or never hormone user), sleep apnoea (ever-diagnosis), snoring (most nights, some nights or never), antidepressant use, smoking status (current, former or never), alcohol consumption in quintiles, diet quality (AHEI-2010) when the dependent variable is physical activity, and physical activity (MET h/week) when the dependent variable is diet quality

${ }^{c}$ Additionally adjusted for quintile of change in AHEI-2010 and MET h/week in 1986

${ }^{\mathrm{d}}$ Additionally adjusted for BMI $\left(<20.9,21-24.9,25-29.9,30.0-31.9,32.0+\mathrm{kg} / \mathrm{m}^{2}\right)$ in 1986

${ }^{\mathrm{e}}$ Additionally adjusted for change in high cholesterol or hypertension, menopausal, snoring, antidepressant use, smoking and alcohol use, AHEI-2010 and MET h/week from 1986 to 2000

${ }^{\mathrm{f}}$ Additionally adjusted for quintile of change in BMI from 1986 to 2000

duration were non-significant. As in the main analysis, only increases of $\geq 2 \mathrm{~h}$ /day were associated with higher risk of diabetes.

\section{Discussion}

This study of 59,031 women is one of the first and the single largest to assess the relationship of long-term changes in selfreported sleep duration with changes in energy balance factors and subsequent risk of diabetes. Extreme increases in sleep duration ( $\geq 2 \mathrm{~h} /$ day) and consistent short sleep ( $\leq 6 \mathrm{~h} /$ day) were adversely associated with diabetes. After BMI adjustment, associations with diabetes were attenuated but remained statistically significant for increases in sleep duration; however, associations with chronic short sleep were attenuated. We also detected modest, adverse associations of 14 year changes in sleep duration with changes in diet quality and physical activity; however, diet quality and physical activity did not appear to mediate the association of increases in sleep duration with diabetes.

Whitehall II is the only other study to examine changes in sleep duration (over $\sim 5$ years) and subsequent diabetes [25]. Although key confounders and intermediates including shift work, sleep apnoea, snoring, depression, diet quality and physical activity were not measured in Whitehall II, the results were strikingly similar to those in our study: compared with consistent $7 \mathrm{~h}$ sleepers, both consistently short sleep $(\leq 5.5 \mathrm{~h} / \mathrm{night}$; OR [95\% CI]: $1.35[1.04,1.76])$ and increases of $\geq 2 \mathrm{~h} /$ night $(1.65[1.15,2.37])$ were adversely associated with diabetes. Similar to our findings, these associations attenuated with adjustment for BMI and change in weight (to $1.25[0.96,1.63]$ and $1.50[1.04,2.16]$, respectively). That the attenuation of chronic short sleep duration with adjustment for BMI and weight change was more pronounced than that for $\geq 2 \mathrm{~h} /$ day increases in both our study and in Whitehall II underscores the possibility that extremes of sleep duration and changes in sleep duration may operate through different mechanisms or reflect different underlying disease processes.

Contrary to our study, most prior longitudinal studies assess whether a single baseline measure of sleep duration predicts future diabetes. Assessing changes in sleep duration is appealing: if achieving a more adequate sleep duration prevented weight gain or diabetes, the corresponding public health message would be to improve sleep duration (e.g. from $\leq 6 \mathrm{~h} /$ day to $7-8 \mathrm{~h} /$ day) to benefit health. However, while chronic short sleep duration was associated with higher risk of diabetes in our study, our results are not consistent with the hypothesis that short or long-sleepers who achieve normative sleep durations will experience benefits for metabolic health. Instead, we found that extreme increases (and not decreases) in sleep duration were associated with weight gain and increased risk of diabetes, independent of prior sleep duration.

One explanation for our finding that increasing sleep duration is adversely associated with diabetes is that age modifies the association of change in sleep with metabolic outcomes. Although we did not detect evidence of interaction, our study included middle-aged and older women; in the younger Quebec family cohort ( $n=216$ adults, $18-64$ years), shortsleepers ( $\leq 6 \mathrm{~h} /$ day) who later slept $7-8 \mathrm{~h} /$ day were less likely to gain weight over 6 years compared with those with chronic 
short sleep [26]. When we conducted a similar analysis, we too found that chronic short-sleepers $(\leq 6 \mathrm{~h} /$ day at both time points) gained more weight compared with women who maintained a normative sleep duration (7-8 h/day). However, in our study, short-sleepers who increased sleep duration to 7-8 h/day had even greater weight gain and higher diabetes risk than those with chronic short sleep, and we found no associations among women with consistently long sleep durations of $\geq 9 \mathrm{~h}$ (although few women followed this pattern).

Our findings are consistent with previous literature on the detrimental impacts of chronic short sleep duration on metabolic health $[5,6,10]$, and newly suggest that the adverse influence of short sleep may not be ameliorated through increases in sleep duration later in life. However, we did not have information on why changes in sleep duration occurred. Changes in sleep duration could reflect voluntary alterations in lifestyle; be mediated through changes in other metabolic, hormonal or behavioural factors associated with diabetes; or represent a distress signal indicating pre-clinical illness. Since we controlled for many indicators of poor health (e.g. diet, physical activity, depression, sleep apnoea, hypercholesterolaemia, hypertension), changes in sleep duration may have a truly bi-directional relationship with changes in weight and metabolic health. In support of the hypothesis that long sleep or extending sleep duration may be truly harmful, in an actigraphy study of healthy adolescents, long sleep remained associated with insulin resistance after BMI adjustment. These findings suggest chronic illness alone does not explain associations of long sleep with metabolic dysregulation (among adolescents, reverse causation is less likely) [27].

Increases in sleep duration can both result from or induce an inflammatory state. For example, proinflammatory cytokines - abundant in obesity - can induce sleepiness [28], while a recent experiment extending time in bed increased inflammation and worsened mood among healthy volunteers [29]. Not only is longer time in bed associated with next-day lethargy and daytime sleepiness, as time asleep and time in bed increase, sleep becomes increasingly fragmented and the total amount of slow wave sleep (particularly restorative) does not increase proportionally [30]. In addition, increases in sleep duration are accompanied by longer periods in the dark, which could be interpreted physiologically as a shorter day-length, previously associated with increased mortality [31]. Thus, extending time in bed may not be a panacea for the adverse influence of short sleep duration on metabolic health.

With respect to the relationship of changes in sleep duration to changes in lifestyle including diet and physical activity, cross-sectional studies suggest associations of both short and long sleep duration with less favourable diet and physical activity [32, 33]. However, prospective evidence is limited and whether long-term changes in sleep duration are accompanied by changes in diet quality or physical activity has not been examined, making few studies directly comparable with ours [34]. In research examining mediation, diet and physical activity did not explain or attenuate the magnitude of associations between baseline sleep duration and weight gain or diabetes [10]. This is consistent with our finding that changes in diet quality and physical activity do not explain associations of change in sleep duration with subsequent diabetes. Weight gain operates differently: in both our study and in Whitehall II [25], weight gain explained the relationship between chronic short sleep duration and diabetes but not the relationship between increases of $\geq 2 \mathrm{~h} /$ day and diabetes. One potential explanation is that measurement error masks mediation by diet and activity but is less of a concern for weight assessment. Another explanation is that chronic short sleep duration influences metabolic health independent of changes in lifestyle; e.g. sleep deprivation increases inflammation [35] or extends light exposure, leading to repressed melatonin secretion, which is associated with diabetes in this cohort [36]. Increases in sleep duration may act on diabetes through other pathways or represent a different phenotype entirely.

The sample size, longitudinal design, long follow-up and repeated measurement enabled examination of changes in sleep duration and of diabetes as an endpoint. These represent important strengths of our study, which is the largest to date and the only study to examine whether changes in diet and physical activity mediate associations of changes in sleep duration with diabetes. Further, we controlled for underlying illness more robustly than prior studies by adjusting for sleep apnoea, snoring, shift work, depression, diet, physical activity, hypercholesterolaemia and hypertension, and by excluding participants with prevalent chronic disease in sensitivity analyses.

Observational research cannot establish causality. An additional limitation was that sleep duration was self-reported and assessed at two time points; fluctuations over follow-up would not be captured. Further, random error in self-reported sleep, diet or activity is magnified when computing change and could attenuate observed associations towards the null. Although self-reported sleep duration in the NHS was validated among 260 participants in 2002 and found to correlate well $(r=0.79)$ with 6 day diaries and have good reproducibility over 2 years [37], no objective measurements distinguishing time in bed from time asleep were taken and napping was not assessed. Thus, we cannot determine to what extent increased sleep duration represents increased time in bed, nor separate daytime from night-time sleep. Although we confirmed selfreported diabetes in medical records, misclassification is possible. However, in a sub-study assessing prevalence of undiagnosed-diabetes, only 1 of 200 women without a previous diagnosis had fasting plasma glucose or fructosamine concentration in the diabetic range [38]. Further, results were similar when excluding cases in the first 2 years, suggesting limited bias from reverse causation. Finally, we examined overall diet quality and weekly physical activity; the circadian timing of 
sleep, meals and activity - not measured here-may also make important contributions to diabetes risk.

Conclusion Chronic short sleep duration and increases in sleep duration are associated with increased risk of diabetes. Decreases in sleep duration have modest, adverse associations with diet quality and physical activity, while increases in sleep duration have modest, adverse associations with weight gain. Ongoing trials will provide further insight as to whether changes in sleep duration influence energy balance. A study among chronically short-sleeping, obese adults could provide insight about the benefits of intentionally extending sleep duration [39], while another study will test whether moderate sleep restriction in long-sleeping older adults improves glucose tolerance and inflammation [8]. As a complement to the forthcoming results of these intervention trials, our study examines longer-term changes in sleep duration with greater duration of follow-up in which diabetes outcomes can be assessed.

Acknowledgements We are indebted to the participants in the NHS for their continuing outstanding support and colleagues working in this study for their valuable help.

Funding This work is supported by National Institute of Health (NIH) grants UM1CA186107, NCI U54CA116847 and NIDDK T32 DK007703.

Duality of interest The authors declare that there is no duality of interest associated with this manuscript.

Contribution statement All authors critically revised the manuscript for important intellectual content and approved the final version. EMC had full access to all the data, analysed the data, wrote and revised the manuscript, and is responsible for the integrity of the work as a whole. EMC, FBH, BR and SR conceived and designed the study question. SNB and $\mathrm{YL}$ assisted in analysing and interpreting the data.

\section{References}

1. International Diabetes Federation (2013) IDF Diabetes Atlas. Available from www.idf.org/diabetesatlas, accessed 5 November 2014

2. Schmid SM, Hallschmid M, Schultes B (2014) The metabolic burden of sleep loss. Lancet Diab Endocrinol 3:52-62

3. Shan Z, Ma H, Xie M et al (2015) Sleep duration and risk of type 2 diabetes: a meta-analysis of prospective studies. Diabetes Care 38: 529-537

4. Yaggi HK, Araujo AB, McKinlay JB (2006) Sleep duration as a risk factor for the development of type 2 diabetes. Diabetes Care 29: $657-661$

5. Spiegel K, Leproult R, Van Cauter E (1999) Impact of sleep debt on metabolic and endocrine function. Lancet 354:1435-1439

6. Lucassen EA, Rother KI, Cizza G (2012) Interacting epidemics? Sleep curtailment, insulin resistance, and obesity. Ann N Y Acad Sci 1264: 110-134
7. Gottlieb DJ, Punjabi NM, Newman AB et al (2005) Association of sleep time with diabetes mellitus and impaired glucose tolerance. Arch Intern Med 165:863-867

8. Youngstedt SD, Jean-Louis G, Bootzin RR et al (2013) Chronic moderate sleep restriction in older long-sleepers and older average duration sleepers: a randomized controlled trial. Contemp Clin Trials $36: 175-186$

9. Bliwise DL, Young TB (2007) The parable of parabola: what the Ushaped curve can and cannot tell us about sleep. Sleep 30:1614 1615

10. Patel SR, Hu FB (2008) Short sleep duration and weight gain: a systematic review. Obesity (Silver Spring, Md) 16:643-653

11. Spiegel K, Leproult R, L'Hermite-Baleriaux M, Copinschi G, Penev PD, Van Cauter E (2004) Leptin levels are dependent on sleep duration: relationships with sympathovagal balance, carbohydrate regulation, cortisol, and thyrotropin. J Clin Endocrinol Metab 89: 5762-5771

12. Spiegel K, Tasali E, Penev P, Van Cauter E (2004) Brief communication: sleep curtailment in healthy young men is associated with decreased leptin levels, elevated ghrelin levels, and increased hunger and appetite. Ann Intern Med 141:846-850

13. St-Onge MP, Roberts AL, Chen J et al (2011) Short sleep duration increases energy intakes but does not change energy expenditure in normal-weight individuals. Am J Clin Nutr 94:410-416

14. Chaput JP (2014) Sleep patterns, diet quality and energy balance. Physiol Behav 134:86-91

15. Chaput JP, St-Onge MP (2014) Increased food intake by insufficient sleep in humans: are we jumping the gun on the hormonal explanation? Front Endocrinol 5:116

16. Klingenberg L, Sjodin A, Holmback U, Astrup A, Chaput JP (2012) Short sleep duration and its association with energy metabolism. Obesity Rev 13:565-577

17. Ayas NT, White DP, Al-Delaimy WK et al (2003) A prospective study of self-reported sleep duration and incident diabetes in women. Diabetes Care 26:380-384

18. Al-Delaimy WK, Manson JE, Willett WC, Stampfer MJ, Hu FB (2002) Snoring as a risk factor for type II diabetes mellitus: a prospective study. Am J Epidemiol 155:387-393

19. Pan A, Schernhammer ES, Sun Q, Hu FB (2011) Rotating night shift work and risk of type 2 diabetes: two prospective cohort studies in women. PLoS Med 8:e1001141

20. Manson JE, Rimm EB, Stampfer MJ et al (1991) Physical activity and incidence of non-insulin-dependent diabetes mellitus in women. Lancet 338:774-778

21. Manson JE, Willett WC, Stampfer MJ et al (1995) Body weight and mortality among women. N Engl J Med 333:677-685

22. Chiuve SE, Fung TT, Rimm EB et al (2012) Alternative dietary indices both strongly predict risk of chronic disease. J Nutr 142: 1009-1018

23. Zhang C, Solomon CG, Manson JE, Hu FB (2006) A prospective study of pregravid physical activity and sedentary behaviors in relation to the risk for gestational diabetes mellitus. Arch Intern Med 166: 543-548

24. Wolf AM, Hunter DJ, Colditz GA et al (1994) Reproducibility and validity of a self-administered physical activity questionnaire. Int J Epidemiol 23:991-999

25. Ferrie JE, Kivimaki M, Akbaraly TN et al (2015) Change in sleep duration and type 2 diabetes: the Whitehall II study. Diabetes Care 38:1467-1472

26. Chaput JP, Despres JP, Bouchard C, Tremblay A (2012) Longer sleep duration associates with lower adiposity gain in adult shortsleepers. Int J Obes (2005) 36:752-756

27. Javaheri S, Storfer-Isser A, Rosen CL, Redline S (2011) Association of short and long sleep durations with insulin sensitivity in adolescents. J Pediatr 158:617-623 
28. Papanicolaou DA, Wilder RL, Manolagas SC, Chrousos GP (1998) The pathophysiologic roles of interleukin-6 in human disease. Ann Intern Med 128:127-137

29. Reynold AM, Bowles ER, Saxena A, Fayad R, Youngstedt SD (2014) Negative effects of time-in-bed extension: a pilot study. J Sleep Med Disord 1:1002

30. Webb WB, Agnew HW Jr (1970) Sleep stage characteristics of long and short-sleepers. Science (New York, NY) 168:146-147

31. Youngstedt SD, Kripke DF (2004) Long sleep and mortality: rationale for sleep restriction. Sleep Med Rev 8:159-174

32. Patel SR, Malhotra A, Gottlieb DJ, White DP, Hu FB (2006) Correlates of long sleep duration. Sleep 29:881-889

33. Stranges S, Dorn JM, Shipley MJ et al (2008) Correlates of short and long sleep duration: a cross-cultural comparison between the United Kingdom and the United States: the Whitehall II Study and the Western New York Health Study. Am J Epidemiol 168:13531364
34. Chaput JP (2014) Sleep patterns, diet quality and energy balance. Physiol Behav 134:86-91

35. Vgontzas AN, Zoumakis E, Bixler EO et al (2004) Adverse effects of modest sleep restriction on sleepiness, performance, and inflammatory cytokines. J Clin Endocrinol Metab 89:2119-2126

36. McMullan CJ, Schernhammer ES, Rimm EB, Hu FB, Forman JP (2013) Melatonin secretion and the incidence of type 2 diabetes. JAMA 309:1388-1396

37. Patel SR, Ayas NT, Malhotra MR et al (2004) A prospective study of sleep duration and mortality risk in women. Sleep 27: 440-444

38. Field AE, Coakley EH, Must A et al (2001) Impact of overweight on the risk of developing common chronic diseases during a 10year period. Arch Intern Med 161:1581-1586

39. Cizza G, Marincola P, Mattingly M et al (2010) Treatment of obesity with extension of sleep duration: a randomized, prospective, controlled trial. Clin Trials (London, England) 7:274-285 